\title{
Ein Stoffwechselkäfig für Versuche mit Ratten
}

\author{
Von \\ Georg Otto SchuÜtz \\ Aus dem Institut für Chemische und Vegetative Pbysiologie der Universität von Nord-Sumatra, Medan, Sumatra, Indonesien \\ (Direktor: Prof. Dr. G. O. Schlütz)
}

(Der Schriftleitung zugegangen am 21. August 1963)

\begin{abstract}
Es wird ein Stoffwechselkäfig für Ratten beschrieben, der es erlaubt, Harn und Kot ohne Beimischung von Futter und Trinkwasser sauber und quantitativ zu bestimmen, und zwar bei exakter Kontrolle der "wahren" Nahrungs- und Flüssigkeitsaufnahme.
\end{abstract}

A metabolic cage for rats is described. Urine and faeces can be cleanly and quantitatively measured without contamination by food or drinking water. There is an exakt check on the true intake of food and fluid.

Nach wie vor stellt die Konstruktion eines guten Stoffwechselkäfigs mit selektiver, quantitativer Auffangung von sauberem Kot und Harn bei gleichzeitiger quantitativer Erfassung von zugeführter Nahrung und Flüssigkeit ein besonderes Problem dar, wie es auch aus der kürzlich erschienen Arbeit von SincLarR und Evans (1) hervorgeht, die einen neuen Stoffwechselkäfig für Versuche mit Nerzen beschrieben haben. -

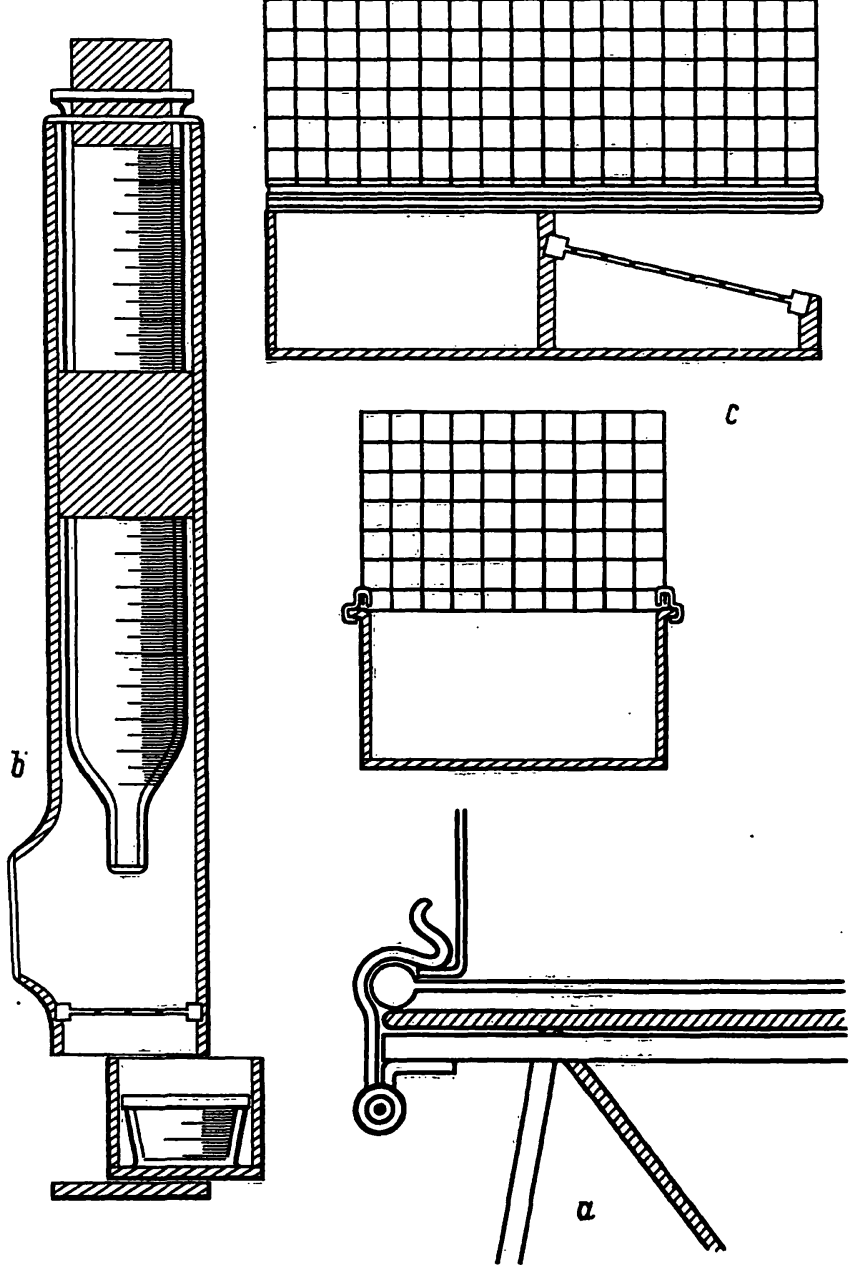

Abb. 1
Bei der Bedeutung, die heute dem Kleintierstoffwechselversuch zukommt, und gestützt auf die Vielfalt der Erfahrungen aus eigenen Versuchen möchten wir einen Stoffwechselkäfig beschreiben, der sich uns sehr gut bewährt hat. Als Grundlage benutzten wir einen Käfig, wie er in den Forschungslaboratorien der HoffmannLa Roche A. G., Basel, verwandt wird ${ }^{1}$ ), und den wir weiter ausbauten und verbesserten.

Auf einem $15 \mathrm{~mm}$ breitem Ringe von $230 \mathrm{~mm}$ Innendurchmesser eines Dreifußes von $550 \mathrm{~mm}$ Höhe ruht ein Plastiktrichter gleichen Durchmessers mit $15 \mathrm{~mm}$ angepreßtem Rande. In dem $45 \mathrm{~mm}$ langen Trichterauslauf von $25 \mathrm{~mm}$ Durchmesser ist ein Messingkonus (Abb. 2) angebracht mit einer Federhalteklammer

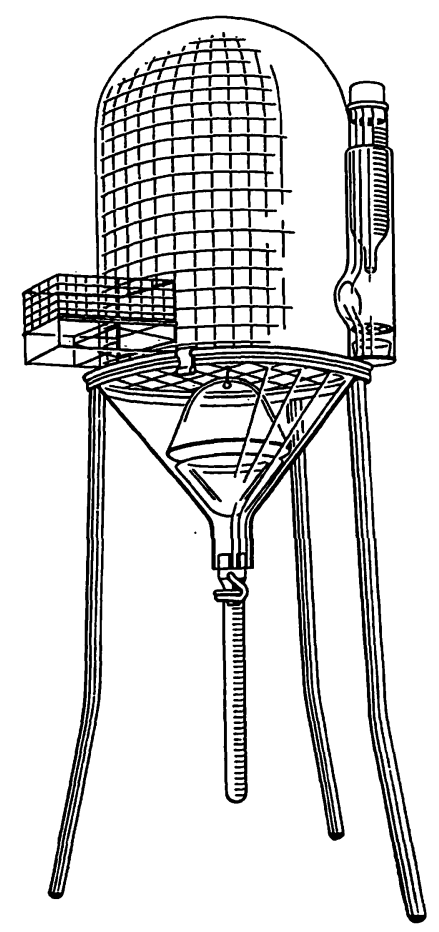

Abb. 2

1) Der Autor möchte an dieser Stelle Herrn Prof. Dr. O. Wrss für seine Unterstützung seinen besonderen Dank aussprechen. 
für ein großes graduiertes Reagenzglas zum Harnauffangen. Auf dem Trichtergrunde ruht eine Preßkalotte mit $45 \mathrm{~mm}$ Innendurchmesser und $40 \mathrm{~mm}$ Höhe sowie einem Kalottendurchmesser von $28 \mathrm{~mm}$. Die Kalotte trägt an den Seiten in je $90^{\circ}$ Abstand vier aufgesetzte Preßstäbe von den Dimensionen $2 \times 3 \times 30 \mathrm{~mm}$, die die Kalotte exakt $2 \mathrm{~mm}$ von der Trichterwand abhalten und somit ein ungehindertes Abfließen des Harnes in das Urinmeßgefäß gestatten, während die Kalotte selbst zum Auffangen des Kotes bestimmt ist (Abb. 2). - Auf dem Trichterrand liegt ein $5 \mathrm{~mm}$ starker Drahtring, auf den ein Drahtnetz von $12 \mathrm{~mm}$ Maschenweite aufgelötet ist, und das den Boden des Stoffwechselkäfigs darstellt. Unter diesem hängt mittels einer Hakenöse in der Mitte des Drahtbodens angebracht eine zweite, durchsichtige Preßkalotte von $92 \mathrm{~mm}$ lichtem Durchmesser und $65 \mathrm{~mm}$ Höhe sowie einem oberen Kalottendurchmesser von $35 \mathrm{~mm}$. Diese Kalotte überdeckt die untere bei weitem und dient zum Trennen von Harn und Kot (Abb. 2). Der Kot fällt auf die Kalotte oder die Trichterwand und gleitet herunter, fällt aber in die untere Kalotte, da er nicht den schmalen Raum zwischen Trichterwand und unterer Kalotte passieren kann. Er sammelt sich daher kontinuierlich in der unteren Kalotte an. Dagegen rinnt der Harn an der Trichterwand - auch derjenige, der über die obere Kalotte fließt - ungehindert durch den schmalen Raum zwischen unterer Kalotte und Trichterwand in das graduierte Harnauffanggefäß, wo seine Menge exakt und mühelos abgelesen werden kann.

Auf dem soeben beschriebenen Bodennetz befindet sich eine $400 \mathrm{~mm}$ hohe Drahtglocke aus $3 \mathrm{~mm}$ weitem Maschendraht von $210 \mathrm{~mm}$ Innendurchmesser und entsprechendem Fußring. Dreifußring, Stoffwechselbodennetz und Drahtglocke werden mit drei kräftigen Federklappklammern zu einer stabilen Versuchseinheit fest zusammengehalten (Abb. 2 u. 1a). An den Seiten der Glocke sind zur Verhinderung des Mischens der Exkrete mit Futter und Wasser die Vorrichtungen für Fliissigkeits- und Nabrungszufubr gesondert ange- bracht (Abb. 1, b u. c). Abbildung 1b zeigt im einzelnen die Flüssigkeitszufubr mit einem $40 \mathrm{~m} l$ fassenden graduierten, gläsernen Wasserbehälter, der sich in einer durchsichtigen Kunststoffröhre mit einer ovalen, in die Glocke hineinragenden Öffnung befindet, durch die das Versuchistier aus dem Vorratsgefäß die Flüssigkeit saugen kann. Abtropfendes, nicht konsumiertes Wasser wird im graduierten Auffanggefäß gesammelt, das gegen Trinkmöglichkeit des Versuchstieres durch ein Drahtnetz abgetrennt wird. Das graduierte Abtropfgefä $B$ kann bequem in das Rohrende eingeschoben und herausgezogen werden. Durch diese Anordnung ist es möglich genau das wahre getrunkene Wasservolumen in der Zeiteinheit $\mathrm{zu}$ messen (Abb. 1 u. 2).

In einem Winkel von $120^{\circ}$ von der Trinkvorrichtung ist die Futteranlage angebracht (Abb. 2 u. 1c). Hier$\mathrm{zu}$ befindet sich in der Drahtglocke eine Öffnung von $50 \times 60 \mathrm{~mm}$ mit verstärktem Rande. Aus deren oberem Teile ragt ein $90 \mathrm{~mm}$ langer Drahtkorb $(50 \times$ $25 \mathrm{~mm}$ ), an dessen unterem Rande sich zwei Nutenschienen zum Einlassen des $90 \mathrm{~mm}$ langen Futternapfes befinden, und der bei $45 \mathrm{~mm}$ Länge unterteilt ist (Abb. 1c). Von der $25 \mathrm{~mm}$ hohen Scheidewand geht bei $20 \mathrm{~mm}$ Höhe ein Drahtgitter ab, das am Anfang des Gefäßes in einer $10 \mathrm{~mm}$ hohen Wand endet. Dieses Drahtnetz dient dazu mitgeschleiftes Futter aus dem Hauptfuttergefä $\beta$ abzufangen, wobei es durch das Gitter in das Verschleppfuttergefäß gerät. Auf diese Weise läßt sich durch einfaches Wägen des ganzen Futtertroges vor und nach dem Versuch das wahre, wirklich aufgenommene Futter rasch bestimmen. - Innerhalb der Drahtglocke befindet sich ein $90 \mathrm{~mm}$ hoher, durchsichtiger Plastikring entsprechenden Durchmessers mit einem Ausschnitt von der Größe des Futterzuganges, der in $10 \mathrm{~mm}$ Bodenhöhe beginnt. (In der Zeichnung nicht dargestellt.) Er dient zum Schließen der Öffnung bei der Entfernung des Futterkastens, was durch einfaches Drehen des Ringes bewerkstelligt wird.

\section{Literatur}

1. Sinclair, D. G. und E. V. Evans, Canad. of Biochem. Physiol. 40, 1395 (1962).

Professor Dr. med. G. O. Schlütz. Professeur advișeur. Chargé de çours à la Faculté dé Médicine. Institut de Biochemie et Nutrition, Université de Damas.

République Arabe Syrienne.

Ständige Anschrift:

Medizinisch-Diagnostisches

Institut Dr. Schlütz,

78 Freiburg i. $\mathrm{Br}$. 